\title{
ALIMENTOS FUNCIONAIS NA CÁRIE DENTAL
}

\section{FUNCTIONAL FOODS IN DENTAL CARIES}

\author{
Julia das Neves Rodrigues Ferreira ${ }^{1}$, Priscila Luiza Mello², Luiz Eduardo Nunes Ferreira ${ }^{3}$
}

\section{RESUMO}

A cárie dental é uma doença multifatorial resultante da interação entre microrganismos, carboidratos ingeridos na dieta e a superfície dental, o que leva ao acúmulo de biofilme. A presença desses microrganismos promove a desmineralização do dente, podendo evoluir para abcessos além de causar dor e consequente comprometimento da qualidade de vida. No controle da cárie dental destaca-se o uso do flúor e clorexidina, porém muitos agentes naturais têm sido testados como alternativas, dentre os quais podemos destacar alguns alimentos funcionais, que além de promover a nutrição básica também propiciam benefícios a saúde. Pesquisas in vitro e in vivo utilizando frutas e seus metabólitos, além de outros componentes da alimentação humana como o chá, têm demonstrado importante potencial anti-cariogênico a ser explorado. Estes alimentos e seus metabólitos promovem benefícios a saúde oral agindo principalmente sobre a inibição do crescimento de bactérias cariogências, inibição da adesão bacteriana, redução da produção de açucares insolúveis e inibição das GTFs. Muitos destes efeitos exercidos sobre $S$. mutans e outras bactérias cariogênicas tem sido atribuído a presença de polifenóis e ácidos orgânicos. Todavia, apesar dos resultados promissores obtidos nas pesquisas laboratoriais, a transposição para humanos tem encontrado muita dificuldade. A complexidade do ambiente oral humano, a forma de preparação das soluções e o tempo de contato dos agentes anti-cariogêncios com biofilmes orais ainda são desafios a serem superados.

PALAVRAS-CHAVE: Alimentos funcionais. Cárie dental. Polifenóis. Biofilme.

\section{ABSTRACT}

Dental caries is a multifactorial disease resulting from the interaction between microorganisms, carbohydrates ingested in the diet and the dental surface, which leads to the accumulation of biofilm. The presence of these microorganisms promotes tooth demineralization, and may evolve to abscesses besides causing pain and consequent impairment of quality of life. In the control of dental caries, the use of fluoride and chlorhexidine stands out, but many natural agents have been tested as alternatives, among which we can highlight some functional foods, which in addition to promoting basic nutrition also provide health benefits. In vitro and in vivo research using fruits and their metabolites, in addition to other components of human food such as tea, have demonstrated important anti-cariogenic potential to be explored. These foods and their metabolites promote oral health benefits by acting primarily on inhibiting the growth of cariogenence bacteria, inhibiting bacterial adhesion, reducing the production of insoluble sugars and inhibiting GTFs. Many of these effects exerted on S. mutans and other cariogenic bacteria have been attributed to the presence of polyphenols and organic acids. However, despite the promising results obtained in laboratory research, transposition to humans has encountered great difficulty. The complexity of the human oral environment, the way solutions are prepared and the contact time of anti-cariogencious agents with oral biofilms are still challenges to be overcome.

KEYWORDS: Functional foods. Dental caries. Polyphenols. Biofilm.

\footnotetext{
${ }^{1}$ Laboratório de Inflamação e Imunologia, Universidade Guarulhos, Guarulhos, SP, Brasil

2 Laboratório de Inflamação e Imunologia, Universidade Guarulhos, Guarulhos, SP, Brasil

3 Laboratório de Inflamação e Imunologia, Universidade Guarulhos, Guarulhos, SP, Brasil. Professor no Programa de Pós-Graduação Stricto Sensu da Universus Veritas Universidade Guarulhos (UNG). Atua como professor em diversos cursos de Graduação nas áreas da Saúde e Biológicas.
} 


\section{RECISATEC - REVISTA CIENTIFICA SAÚDE E TECNOLOGIA}

ALIMENTOS FUNCIONAIS NA CÁRIE DENTAL Julia das Neves Rodrigues Ferreira, Priscila Luiza Mello, Luiz Eduardo Nunes Ferreira

\section{INTRODUÇÃO}

A cárie dental representa um grande problema de saúde coletiva bucal (Dinelli et al., 2000; Oliveira et al., 2006), tanto no Brasil (Dantas et al., 2000; Brasil, 1988, 1996) como na maior parte do mundo (Loretto et al., 2000; Martins et al., 1999; Weyne, 1997). Esses achados são confirmados pelos resultados do último Saúde Bucal (SB) Brasil (Brasil, 2010), que mostram que, apesar de uma melhoria no estado de saúde bucal dos brasileiros, a cárie continua sendo o principal problema de saúde bucal que os acomete.

Essa infecção multifatorial é causada pelo acúmulo de biofilme na superfície do dente e assim como outras doenças bucais, não representa uma ameaça à vida, mas pode afetar a qualidade de vida de muitos indivíduos, uma vez que pode causar dor e vir acompanhada de limitações funcionais e sociais (Keyes, 1962; Socransky, 1972; Marsh, 2003). A cárie dentária, se não tratada inicialmente pode levar a uma condição de dor podendo ter consequências na alimentação, tanto na qualidade como na quantidade, e pode também acometer a saúde em outros aspectos como, fonação, deglutição e mastigação (Chaves et al., 2001).

O não tratamento pode progredir para a formação de abscessos, onde a ocorrência na dentição decídua pode afetar o desenvolvimento da dentição permanente, porém o aspecto mais preocupante relacionado à formação de abscessos é o risco de ocorrer uma septicemia (Billings, 1996). Dessa maneira, torna-se importante avaliar o impacto da cárie na qualidade de vida de uma criança, uma vez que reflete a autopercepção sobre a saúde e as consequências da saúde bucal na sua vida (Low \& Tan 1999; Ribeiro et al., 2004).

\section{DESENVOLVIMENTO DA CÁRIE}

A cárie é o resultado de uma interação entre microrganismos (biofilme), fatores da dieta e sua fermentação sobre o dente, caracterizada pelo acúmulo de biofilme na superfície dental (Marsh, 2003), relacionado a um consumo frequente de carboidratos fermentáveis (Bowen et al., 1980) que possibilitam um aumento no crescimento de bactérias cariogênicas, altamente eficientes na conversão de carboidratos a ácidos orgânicos, que têm a capacidade de desmineralizar o esmalte do dente (Lemos \& Burne, 2008). Quando ocorrem alterações no pH da matriz do biofilme, causados pela dieta, microrganismos ou fluxo e/ou componentes salivares há um desequilíbrio entre o biofilme e o hospedeiro o que leva ao aparecimento de doenças como a cárie, cujo principal agente etiológico é o Streptococcus mutans.

Pela teoria da "placa específica" certos tipos de placas bacterianas (ou biofilmes bacterianos) são consideradas odontopatogênicas, pois são colonizadas por bactérias capazes de determinar uma quantidade mensurável de cárie dentária. Estas espécies odontopatogênicas incluem S. mutans e Lactobacillus que são responsáveis por boa parte das lesões de cárie, 


\section{RECISATEC - REVISTA CIENTÍFICA SAÚDE E TECNOLOGIA}

ALIMENTOS FUNCIONAIS NA CÁRIE DENTAL Julia das Neves Rodrigues Ferreira, Priscila Luiza Mello, Luiz Eduardo Nunes Ferreira

embora seja possível a ocorrência de placa sem que haja doença, significando a presença de placa não dominada pelos organismos odontopatógenos (Marinho \& Pereira, 1998).

Segundo Koo et al. (2002) a placa dental é formada principalmente em dois estágios: adesão inicial reversível de várias bactérias orais na superfície do dente, seguido pelos dependentes de sucrose onde pela atividade das glicosiltransferases (GTFs) sintetizam camadas de glucanos insolúveis, onde ocorre uma firme e irreversível adesão do $S$. mutans.

Os microrganismos considerados cariogênicos são capazes de se aderir à superfície dental e causar a sua desmineralização produzindo ácidos orgânicos fracos a partir da sacarose (acidogênicos) e manterem o seu metabolismo mesmo em pHs baixos (acidúricos), formam polissacarídeos intra e extracelulares de reserva e extracelulares insolúveis, que são responsáveis pela adesividade da placa bacteriana (Yoshizumi et al., 2007; Loesche, 1986; Macpherson et al., 1992). Os dentes também variam em susceptibilidade a caries de acordo com a composição, morfologia, locação e posição, a qual pode promover a retenção da placa (Loveren et al., 2012).

Dessa forma, a virulência de $S$. mutans está diretamente relacionada com a sua habilidade de: 1) formar biofilme que adere na superfície do dente; 2) produzir grandes quantidades de ácidos orgânicos a partir de carboidratos ingeridos na dieta do hospedeiro (acidogenicidade), e 3) tolerar o estresse ambiental, principalmente pela sobrevivência em meio ácido (aciduricidade) (Quivey et al., 2000; Bowen, 2002; Lemos et al., 2005).

\section{TRATAMENTO DA CÁRIE}

Sabe-se que existem muitas maneiras para tentar prevenir e controlar a cárie dental, com especial destaque ao uso do flúor e da clorexidina e à associação de ambas (Souza, 2002), mas apesar disso a cárie continua sendo dentro da odontologia a doença de maior prevalência.

Atualmente o antisséptico sintético clorexidina é geralmente aceito como agente mais eficiente para a prevenção ou tratamento de doenças orais. Porém, o uso rotineiro de clorexidina não é recomendado devido aos seus efeitos colaterais locais, particularmente manchas nos dentes e formação de cálculos (Sköld-Larsson et. al., 2004; Charles et al., 2004; Paraskevas, 2005). Muitas pesquisas têm sido desenvolvidas visando detectar novos agentes naturais capazes de reduzir ou prevenir cáries como uma forma alternativa aos tratamentos convencionais (Gazzani et. al., 2012) e dentre os produtos naturais destacamos extrato de plantas, óleos essenciais e a própolis (Duarte et. al., 2006; Galvão et al., 2012; Groppo et. al. 2002).

Recentemente o surgimento de resistência dos patógenos aos agentes antibacterianos convencionais e a necessidade de desenvolver novas estratégias para o controle de doenças infecciosas levou a uma intensa pesquisa sobre produtos naturais capazes de agir como agentes antimicrobianos. Essas pesquisas têm levado a descobertas de novos compostos presentes em diversos vegetais e fungos, capazes de agir por diferentes mecanismos contra os principais agentes causadores de doenças orais (Ooshima et al., 1998; Hamilton-Miller, 2001). 


\section{RECISATEC - REVISTA CIENTÍFICA SAÚDE E TECNOLOGIA}

ALIMENTOS FUNCIONAIS NA CÁRIE DENTAL Julia das Neves Rodrigues Ferreira, Priscila Luiza Mello, Luiz Eduardo Nunes Ferreira

Diante disso, novas tendências para o controle de doenças orais, como a cárie dental, devem levar em consideração a importância do equilíbrio entre patógenos e comensais, sendo importante o desenvolvimento de alternativas que promovam a inibição desses patógenos, modulando o balanço da microbiota. Dentre estas novas tendências, a utilização de produtos probióticos com ação oral é uma alternativa promissora uma vez que pode-se comprovar os benefícios destes à saúde de um modo geral (Favaro-Trindade et al., 2011).

\section{ALIMENTOS FUNCIONAIS}

Durante as últimas décadas, uma relação entre dieta e saúde tem sido apontada através das descobertas de um grande número de investigações epidemiológicas. Através dessas descobertas, atualmente, alimentos não são mais considerados somente pelo seu valor nutritivo, mas também pelos seus potenciais efeitos positivos em prevenir e proteger contra diversas doenças. Na odontologia está bem estabelecido que a prática de colocar flúor na água, o sal e o leite são capazes de prevenir caries dentais. O uso de outros alimentos para promover benefícios a saúde oral tem se mostrado promissor a medida que as pesquisas avançam (Daglia et al., 2011; Loveren et al., 2012).

A International Food Information Concil (IFIC) definiu alimentos funcionais como: "alimentos que promovem um benefício a saúde além da nutrição básica". Desta forma, os alimentos ou componentes de alimentos que produzem efeitos benéficos peculiares a saúde humana, além da nutrição básica, é definida como alimentos funcionais (Daglia et al., 2011).

\section{POLIFENÓIS}

Os benefícios dos polifenóis presentes na dieta para a saúde têm chamado a atenção dos nutricionistas nos últimos anos. Os polifenóis têm apresentado ação antioxidante, anticâncer e anti-inflamatória, além da prevenção de doenças cardiovasculares através da inibição da oxidação de lipoproteínas de baixa densidade (Scalbert et. al., 2005; Block et al., 1992; Marrugat et. al., 2004, Ruf, 2003).

Frutas comestíveis são uma rica fonte de polifenóis como os ácidos fenólicos e flavonóides (Yanagida et al., 2000). Nas últimas décadas muitos estudos sobre os efeitos anticariogênicos dos extratos de polifenóis de diferentes plantas tem sido reportados (Ito et al., 1984, Kakiuchi et al., 1986; Sawamura et al., 1992; Tagashira et al., 1997; Mitsunaga \& Abe, 1997), o qual pode estar relacionado a suas atividades antioxidantes (Loveren et al., 2012).

Extrato de plantas contendo altos níveis de polifenóis inibem o crescimento de $S$. mutans e outras bactérias. A inibição do $S$. mutans ocorre na presença de glicose e sucrose, dois dos principais componentes usados em alimentos. A produção de ácidos e adesão de $S$. mutans também é reduzida na presença dos polifenóis (Smullen et al., 2006). Estes compostos formam complexos com proteínas e polissacarídeos (Haslan et al., 1996), sendo provável que polifenóis polimerizados se liguem a componentes da superfície celular, ao invés de penetrar nas células, 
inibindo enzimas extracelulares ou enzimas de adesão celular. Polifenóis também se ligam a íons metálicos e a redução dos íons de metal por complexação com polifenóis podem inibir o crescimento celular. Os grandes polímeros de procyanidin podem apresentar mais locais para quelação de metais, resultando em uma maior atividade de inibição de crescimento (Scalbert, 1991; Smullen et al. 2006).

Outras substâncias identificadas com atividade antimicrobiana contra patógenos orais são o ácido oleanólico, oleanolic aldehyde, linoleic acid, betulinic acid, 5 (hydroxymetyl) 2-furfural, rutin, beta-sitosterol e beta-sitosterol glucoside (Loveren et al., 2012).

\section{MAÇÃ}

O consumo de maçã tem sido relacionado a uma boa saúde dental (Bibby, 1983). Efeitos inibitórios de polifenóis da maça sobre a síntese dos glucanos insolúveis em água pela glicosiltransferase dos Streptoccocus do grupo mutans e sobre a aderência das células bacterianas foram demonstrados em estudos in vitro (Gazzani et al., 2012). Polifenóis presentes no extrato da maçã, assim como taninos condensados, marcadamente inibiram a síntese de glucanos insolúveis por $S$. sobrinus e $S$. mutans in vitro. Polifenóis extraídos de maças imaturas inibiram a atividade da GTF bacteriana, assim como a aderência e crescimento de bactérias cariogênicas (Yanagida et al., 2000).

\section{VINHO E UVAS}

Uvas são uma das mais amplas e economicamente importantes culturas de frutas, sendo principalmente utilizados para fazer vinho (Mazza et al.,1993). As uvas de vinho são um rico recurso de polifenóis com potencial bioativo, caracterizando-se por poder apresentar compostos como flavonóides, antocianinas, proantocianidinas, catechins e outros compostos fenólicos (Kammerer et al., 2004). No entanto, as principais atividades antimicrobianas do vinho se não se devêm à presença de polifenóis, mas sim de ácidos orgânicos de baixo peso molecular que normalmente ocorrem nas uvas ou formados durante o processo de fermentação malolactic (Daglia et al., 2007).

A inibição do $S$. mutans sucrose-dependente, adesão do $S$. mutans a hidroxiapatita sucrose independente e formação do biofilme, assim como um aumento no desprendimento do biofilme da superfície de hidroxiapatita foram detectados quando testado o vinho tinto desalcolizado. A atividade inibitória foi associada as proanthocyanidins e, em menor grau, aos pigmentos vermelhos (Daglia, et al., 2010).

Timothe et al. (2007) testou extratos fenólicos extraídos de diversas variedades de uvas, sobre vários fatores de virulência de $S$. mutans. Apesar dos extratos de uva apresentarem concentrações diferentes de polifenóis, todos os tipos de uvas testadas foram capazes de inibir as GTFs do $S$. mutans, sendo esta uma importante enzima envolvida na produção de polissacarídeos extracelulares e no crescimento do biofilme (Signoretto et al., 2012). Desta forma estes extratos polifenólicos abrigam compostos que podem ser úteis para controle do biofilme, porém não 
exercem efeitos sobre a viabilidade bacteriana, onde os efeitos antibacterianos parecem estar mais relacionados a presença de ácidos orgânicos (Daglia et al., 2007).

As uvas-passa também contêm diversos fitoquímicos com propriedades sobre patógenos orais. Wu (2009) verificou os efeitos de diversos compostos extraídos de uvas passas obtidas de Vitis vinífera L. (Vitacea), contra P. gingivalis e S. mutans. O oleanolic acid, oleanolic aldehyde e 5-(hydroxymethil)-2-furfural exerceram atividade contra $P$. gingivalis. Esses compostos juntamente com o rutin, foram efetivos contra $S$. mutans.

O extrato da semente da uva mostrou-se capaz de contribuir positivamente, prevenindo os processos de desmineralização ou auxiliando na remineralização em lesões de cariei radicular, podendo ser um potencial adjuvante ou uma alternativa para o flúor no tratamento das cáries radiculares durante a terapia invasiva mínima (Wu et al., 2009).

\section{NOZ-MOSCADA}

Myristica fragrans (Myristicaceae), comumente conhecida como noz-moscada, é comumente empregada como tempero em várias partes do mundo. Na medicina popular tem aplicação como remédio contra gases, hipolipidêmico, antitrombótica, contra agregação plaquetária, antifúngico, afrodisíaco, calmante, anti-ulcerogêncio, antitumoral e atividade antiinflamatória (Morita et al., 2003; Sonavane et al., 2002; Capasso et al., 2000; Park et al., 1998; Ozaki et al., 1989).

$O$ arilo de $M$. fragans demonstrou atividade anticariogência contra $S$. mutans (Hattori et al., 1986). Já o composto isolado de $M$. fragans, macelignan, demonstrou uma forte atividade antibacteriana contra os Streptococcus e Lactobacillus spp., podendo efetivamente bloquear a formação de cáries dentais através da prevenção da formação de biofilme e redução do $\mathrm{pH}$. O fato da noz-moscada ser há muito tempo utilizada na alimentação, sendo considerado um alimento seguro, torna possível uma futura aplicação como um novo agente natural na prevenção de cáries dentais, podendo ser empregado como um alimento funcional ou na formulação de novos produtos para a saúde bucal (Chung et al., 2003).

\section{CHÁ}

O chá é uma infusão aquosa das folhas secas da planta Camellia sinensis L. (família Theaceae), este é classificado de acordo com o processo de manufatura, apresentando três principais tipos: chá verde não fermentado, chá oolong semi-fermentado e o chá preto fermentado. Acredita-se que o uso de C. sinensis tem origem a 4.000 anos atrás, sendo atualmente uma das bebidas mais consumidas no mundo. Além disso, é considerado uma bebida segura pela Food and Drug Administration (FDA) nos Estados Unidos (Wu \& Wei, 2002).

O chá corresponde a uma rica fonte de polifenóis e seus níveis variam de acordo com a forma de processamento (Astill et al., 2001). Polifenóis do chá claramente inibem o crescimento de bactérias cariogênicas e a produção dos glucanos insolúveis pela GTFs. O chá verde apresenta uma maior concentração de polifenóis monoméricos como as epicatechin, epicatechin 


\section{RECISATEC - REVISTA CIENTÍFICA SAÚDE E TECNOLOGIA}

ALIMENTOS FUNCIONAIS NA CÁRIE DENTAL Julia das Neves Rodrigues Ferreira, Priscila Luiza Mello, Luiz Eduardo Nunes Ferreira

gallate e epigallocatechin. Durante o processo de fermentação ocorre a oxidação das catechins, levando a formação de polifenóis oligoméricos, estando presentes principalmente no chá preto (Yam et al., 1997). Estudos demonstraram que o epigallocatechin gallate (EGCg) das folhas do chá e alguns polímeros oxidados produzidos pela fermentação são os principais inibidores da GTF (Yanagida et al., 2000).

Testes in vitro utilizando o extrato bruto das folhas de chá verde foram capazes de causar uma significativa inibição na aderência de $S$. mutans em hidroxapatita, além de inibir da atividade da GTF. Nos testes in vivo, o extrato foi capaz de reduzir a formação de caries em ratos (Otake et al., 1991) e em humanos com o uso de enxaguatórios bucais (Ferrazzano et al., 2011).

O efeito inibitório do extrato do chá oolong sobre as propriedades da cárie induzidas por S. mutans foi examinado in vitro. Esse extrato foi capaz de reduzir a taxa de produção de ácidos pelo $S$. mutans, além de diminuir a taxa de crescimento desta bactéria. Por outro lado, os produtos deste chá semi-fermentado reduziram a hidrofobicidade da superfície celular em várias espécies de Estreptococcus (Matsumoto et al., 1999).

Polifenóis isolados do chá semi-fermentado (oolong) apresentaram uma forte ação inibitória sobre a atividade da GTF de $S$. sobrinus. Muitos dos polifenóis não foram encontrados no chá verde, onde a formação destes pode estar relacionado ao processo de fermentação (Nakahara et al., 1993). Frações do chá oolong livre de catechin monoméricas demonstraram uma significativa inibição na síntese de glucanos insolúveis via GTFs. Testes in vivo também reduziram significativamente o índice de cáries e seus escores (Ooshima et al., 1993; Ooshima et al., 1998). Matsumoto et al. (2003) verificou que frações específicas de polifenóis poliméricos presentes no chá semi-fermentado prejudica a síntese de glucanos pela inibição não competitiva, inibindo a região domínio de ligação do glucano a enzima.

Com relação ao chá preto, um estudo verificou a ação do extrato bruto deste chá sobre a formação de cáries em hamsters quando submetidos a uma dieta regular ou cariogência. $O$ consumo de chá preto foi capaz de reduzir a formação de cáries mesmo na presença de açucares na dieta (Linke \& LeGeros, 2003).

Outros experimentos também demonstraram a inibição da atividade da amilase salivar pelos extratos feitos a partir dos chás. Este efeito sobre a amilase salivar também pode contribuir para uma significante redução da cariogenicidade presente nos alimentos que contém amido (Kashket \& Paolino, 1988).

\section{LIMITAÇÕES}

Muitos dos estudos relatam a atividade anticárie por ensaios utilizando $S$. mutans em estado planctônico através dos testes de determinação da concentração inibitória mínima (MIC) e concentração bactericida mínima $(\mathrm{MBC})$. É importante ressaltar que tais experimentos são conduzidos a fim de se detectar a sensibilidade do micro-organismo ao agente testado, levando em conta a condição estática do teste e o tempo de contato entre o agente e a bactéria em uma 


\section{RECISATEC - REVISTA CIENTÍFICA SAÚDE E TECNOLOGIA}

ALIMENTOS FUNCIONAIS NA CÁRIE DENTAL Julia das Neves Rodrigues Ferreira, Priscila Luiza Mello, Luiz Eduardo Nunes Ferreira

condição que não é encontrada naturalmente no ambiente bucal (Simões, 2011). Modelos experimentais utilizando biofilme monoespécie tentam criar uma situação semelhante ao ambiente bucal por incorporar elementos encontrados no biofilme cariogênico, como por exemplo, a matriz rica em polissacarídeos. Apesar disso, como avalia a ação sobre um único micro-organismo, a resposta em um ambiente colonizado por outras espécies e a presença de outros fatores, como por exemplo, enzimas do hospedeiro, poderia alterar o resultado esperado em uma aplicação in situ ou in vivo. Além disso, na maioria dos casos, alimentos e bebidas consumidos tem um tempo curto de contato entre os tecidos orais, o que deve ser levado em consideração durante o delineamento dos estudos (Gazzani et al., 2012).

Em estágios mais avançados sobre o conhecimento da atividade anticárie de certos produtos, estudos envolvendo a formação de cárie em animais, ou avaliação da atividade in situ trariam uma resposta mais próxima do acurada do real potencial uso de um produto no combate a cárie. Após esta etapa, os estudos clínicos - ainda escassos - são os responsáveis por confirmar a possibilidade de se manter o investimento em um produto de fato efetivo e seguro no combate a cárie (Jeon et al., 2011). Talvez pela complexidade que exigem para serem bem conduzidos além de ser naturalmente mais dispendioso seriam entraves para a sua realização com mais frequência, sendo que a descrição de eficácia se limita apenas a relatos empíricos.

\section{CONCLUSÃO}

Muitos alimentos e seus compostos têm apresentado potencial para ser empregado como alimentos funcionais com atividade antimicrobiana ou na formulação de produtos voltados para a saúde oral. Produtos naturais e outros alimentos compõem uma larga fonte de recursos a serem explorado (Jeon et al., 2011). No entanto, muitos estudos realizados in vitro ou in vivo em animais tem tido dificuldades para transpor para humanos devido a inúmeros fatores. Dessa maneira, existe um incentivo na busca por metodologias que possam fornecer resultados que demonstrem o real potencial deste grupo de produtos com reconhecida atividade anticárie.

\section{REFERÊNCIAS BIBLIOGRAFICAS}

1 Astill C, Birch MR, Dacombe C, Humphrey PG, Martin PT: Factors affecting the caffeine and polyphenol contents of black and green tea infusions. J Agric Food Chem 2001; 49: 53405347.

2 Bibby BG. Fruits and vegetables and dental caries. Clin. Prev. Dent. 1983, 5, 3-11.

3 Block G, Patterson B, Subar A. Fruit, vegetables, and cancer prevention: a review of the epidemiological evidence. Nutr Cancer 1992;18(1):1. 
4 Bowen WH, Amsbaugh SM, Monell-Torrens S, Brunelle J, Kuzmiak-JonesH, Cole MF. A method to assess cariogenic potential of foodstuffs. Journal of the American Dental Association,

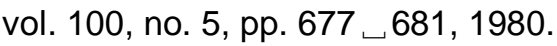

5 Bowen $\mathrm{WH}$. Do we need to be concerned about dental caries in the coming millennium? J Am Dent Assoc. 2002; 133: 1405-7.

6 Brasil. Ministério da Saúde. Departamento de Informática do SUS (DATASUS). Levantamento Epidemiológico em Saúde Bucal - Cárie Dental 1996. Brasília: Ministério da Saúde; 1996 [acesso 2009 Jun 6]. Disponível em: http://tabnet.datasus.gov.br/.

7 Brasil. Ministério da Saúde. Secretaria Nacional de Programas Especiais de Saúde. Divisão Nacional de Saúde Bucal. Fundação Serviços de Saúde Pública. Levantamento epidemiológico em saúde bucal: Brasil, zona urbana, 1986. Brasília: Centro de Documentação do Ministério da Saúde; 1988.

8 Brasil. Ministério da Saúde. Departamento de Atenção Básica. Coordenação Nacional de Saúde Bucal. Projeto SB Brasil 2010 - Pesquisa Nacional de Saúde Bucal. Primeiros Resultados. Brasília; 2011.

9 Capasso R, Pinto L, Vuotto ML, Di Carlo G, 2000. Preventive effect of eugenol on PAF and ethanol-induced gastric mucosal damage. Fitoterapia, 71 (Suppl. 1), S131-S137.

10 Charles $\mathrm{CH}$, Mostler KM, Bartels LL, Mankodi SM. Comparative antiplaque and antigingivitis effectiveness of a chlorhexidine and an essential oil mouthrinse: 6 -month clinical trial. J Clin Periodontol. 2004; 31(10): 878-84.

11 Chaves HCB, Freitas RL, Colares V. Perfil psicosocial dos responsáveis por pré-escolares portadores de cárie de mamadeira da cidade de Recife. In: Anais da Faculdade de Odontologia da Universidade Federal de Pernambuco. Recife. UFPE; 2001. P. 31-7.

12 Chung JY, Choo JH, Lee MH, Hwang JK. Anticariogenic activity of macelignan isolated from Myristica fragrans (nutmeg) against Streptococcus mutans. Phytomedicine. 2003; 13(4): 2616.

13 Daglia M, Papetti A, Grisoli P, Aceti C, Dacarro C, Gazzani G. Antibacterial activity of red and white wine against oral streptococci. Journal of Agricultural and Food Chemistry. 2007; 55 (13): 5038-5042.

14 Daglia M, Stauder M, Papetti A, Signoretto C, Giusto G, Canepari P, Pruzzo C, Gazzani G: Isolation of red wine components with anti-adhesion and anti-biofilm activity against Streptococcus mutans. Food Chem 2010, 119:1182-1188.

15 Daglia M, Papetti A, Mascherpa D, Grisoli P, Giusto G, Lingström P, Pratten J, Signoretto C, Spratt DA, Wilson M, Zaura E, Gazzani G. Plant and fungal food components with potential activity on the development of microbial oral diseases. J Biomed Biotechnol. 2011: 27: 45-78.

16 Dantas S, Oliveira AGRC, Frazäo P, Ely HC, Araújo IC, Pattussi MP. Como está o sorriso do Brasil? Rev ABO Nac. 2000; 8(3): 134-5.

17 Dinelli W, Corona SAM, Dinelli TC, Garcia PPNS. Desenvolvimento, aplicação e avaliação de um programa de orientação sobre higiene bucal junto à pré-escolares. Stoma. 2000; 13(57): 27-30. 


\section{RECISATEC - REVISTA CIENTÍFICA SAÚDE E TECNOLOGIA}

18 Duarte S, Rosalen PL, Hayacibara MF, Cury JA, Bowen WH, Marquis RE, Rehder VL, Sartoratto A, Ikegaki M, Koo H. The influence of a novel propolis on mutans streptococci biofilms and caries development in rats. Arch Oral Biol. 2006; 51(1):15-22.

19 Ferrazzano GF, Roberto L, Amato I, Cantile T, Sangianantoni G, Ingenito A. Antimicrobial properties of green tea extract against cariogenic microflora: an in vivo study. J Med Food. 2011;14: 907-11.

20 Gazzani G, Daglia M, Papetti A. Food components with anticaries activity. Curr Opin Biotechnol. 2012; 23(2):153-9.

21 Galvão LC, Furletti VF, Bersan SM, da Cunha MG, Ruiz AL, de Carvalho JE, Sartoratto A, Rehder VL, Figueira GM, Teixeira Duarte MC, Ikegaki M, de Alencar SM, Rosalen PL. Antimicrobial Activity of Essential Oils against Streptococcus mutans and their Antiproliferative Effects. Evid Based Complement Alternat Med. 2012;2012:751435. Epub 2012 May 24.

22 Groppo FC, Ramacciato JC, Simões RP, Flório FM, Sartoratto A. Antimicrobial activity of garlic, tea tree oil, and chlorhexidine against oral microorganisms. Int Dent J. 2002 Dec; 52(6):4337.

23 Hamilton-Miller JMT. Anti-cariogenic properties of tea (Camellia sinensis). Journal of Medical Microbiology. 2001; 50: 299-302.

24 Haslam E. Natural polyphenols (vegetable tannins) as drugs: possible modes of action. $\mathrm{J}$ Nat Prod. 1996; 59: 205-215.

25 Hattori M, Hada S, Watahiki A, Ihara H, Shu YZ, Kakiuchi N, Mizuno T, Namba, T. Studies on dental caries prevention by traditional medicines. X. Antibacterial action of phenolic components from mace against Streptococcus mutans. Chem. Pharm. Bull. 1986; 34: 3885-3893.

26 Ito $\mathrm{M}$, Uyeda $\mathrm{M}$, Iwatani T, Nakagawa $\mathrm{Y}$. Flavonoids as a possible preventive of dental caries. Agric. Biol. Chem. 1984; 48: 2143-2145.

27 Jeon JG, Rosalen PL, Falsetta ML, Koo H. Natural products in caries research: current (limited) knowledge, challenges and future perspective. Caries Res. 2011; 45: 243-63.

28 Kakiuchi N, Hattori M, Nishizawa M, Yamagishi T, Okuda T, Namba T. Inhibitory effect of various tannins on glucan synthesis by glucosyltransferase from Streptococcus mutans. Chem. Pharm. Bull. 1986; 34: 720-725.

29 Kammerer D, Claus A, Carle R, Schieber A. Polyphenol screening of pomace from red and white grape varieties (Vitis vinifera L.) by HPLC-DAD-MS/MS. J. Agric. Food Chem. 2004; 52: 4360-4367.

30 Kashket S, Paolino VJ. Inhibition of salivary amylase by water-soluble extracts of tea. Arch Oral Biol. 1988; 33(11): 845.

31 Keyes PH. Dental caries in the molar teeth of rats. I.Distribution of lesions induced by high carbohydrate low-fat diets. J Dent Res. 1958; 37(6): 1077-87. Colocar 1958 no texto 


\section{RECISATEC - REVISTA CIENTIFICA SAÚDE E TECNOLOGIA}

ALIMENTOS FUNCIONAIS NA CÁRIE DENTAL Julia das Neves Rodrigues Ferreira, Priscila Luiza Mello, Luiz Eduardo Nunes Ferreira

32 Koo H, Rosalen PL, Cury JA, Park YK, Bowen WH, 2002. Effects of compounds found in propolis on Streptococcus mutans growth and on glucosyltransferase activity. Antimicrob. Agents Chemother. 46, 1302-1309.

33 Lemos JA, Burne RA. A model of efficiency: stress tolerance by Streptococcus mutans. Microbiology. 2008; 154: 3247-55.

34 Lemos JAC, Abranches J, Burne RA. Responses of cariogenic streptococci to environmental stresses. Curr. Issues. Mol. Bio. 2005; 7: 95-108.

35 Linke HA, LeGeros RZ. Black tea extract and dental caries formation in hamsters. Int J Food Sci Nutr. 2003; 54(1):89.

36 Loesche WJ. Role of Streptococcus mutans in human dental decay. Microbiol Rev. 1986; 50(4): 353-80.

37 Loretto NRM, Seixas ZA, Jardim MC, Brito RL. Cárie dentária no Brasil: alguns aspectos sociais, políticos e econômicos. Rev ABO Nac. 2000; 8(1): 45-9.

38 van Loveren C, Broukal Z, Oganessian E. Functional foods/ingredients and dental caries. Eur J Nutr. 2012 Jul;51 Suppl 2:S15-25

39 Low W, Tan S, Scwartz S. The effect of severe caries on the quality of life in young children. Pedriatr Dent. Sept-Oct 1991; 21 (6): 325-6.

40 Macpherson LM, Macfarlane TW, Geddes DAM, Stephen KW. Assessment of the cariogenic potential of Streptococcus mutans strains and its relationship to in vivo caries experience. Oral Microbiol Immunol. 1992; 7(3): 142-7.

41 Marinho VA, Pereira GM. Cárie: diagnóstico e plano de tratamento. Rev Univ Alfenas. 1998; 4: 27-37.

42 Marsh PD. Are dental diseases examples of ecological catastrophes? Microbiology. 2003; 149(Pt 2): 279-94.

43 Martins MD, Araújo RGD, Veloso NF. Avaliação das necessidades de tratamento odontológico de crianças de baixa renda. JBP J Bras Odontopediatr Odontol Bebe. 1999; 2(6): $132-6$.

44 Marrugat J, Covas Ml, Fitó M, Schröder H, Miró-Casas E, Gimeno E, López-Sabater MC, de la Torre R, Farré M. Effects of differing phenolic content in dietary olive oils on lipids and LDL oxidation--a randomized controlled trial. Eur J Nutr. 2004; 43(3):140.

45 Matsumoto M, Minami T, Sasaki H, Sobue S, Hamada S, Ooshima T. Inhibitory effects of oolong tea extract on caries-inducing properties of mutans streptococci. Caries Res 1999; 33(6):441.

46 Matsumoto M, Hamada S, Ooshima T: Molecular analysis of the inhibitory effects of oolong tea polyphenols on glucan-binding domain of recombinant glucosyltransferases from Streptococcus mutans mt8148. FEMS Microbiol. Lett 2003; 228: 73-80. 


\section{RECISATEC - REVISTA CIENTÍFICA SAÚDE E TECNOLOGIA}

ALIMENTOS FUNCIONAIS NA CÁRIE DENTAL Julia das Neves Rodrigues Ferreira, Priscila Luiza Mello, Luiz Eduardo Nunes Ferreira

47 Mazza G, Miniati E. Grapes: In Anthocyanins in Fruits, Vegetables and Eds. CRC Press: Boca Raton, 1993; 149-199.

48 Mitsunaga, T., Abe, I. Inhibitory effects of bark proanthocyanidins on the activities of glucosyltransferases of Streptococcus sobrinus. J. Wood Chem. Technol. 1997; 17: 327-340.

49 Morita $T$, Jinno $K$, Kawagishi $H$, Arimoto $\mathrm{Y}$, Suganuma $\mathrm{H}$, Inakuma $\mathrm{T}$, Sugiyama, $\mathrm{K}$. Hepatoprotective effect of myristicin from nutmeg (Myristica fragrans) on lipopolysaccharide/dgalactosamine-induced liver injury. J. Agric. Food Chem. 2003; 51: 1560-1565.

50 Nakahara K, Kawabata S, Ono H, Ogura K, Tanaka T, Ooshima T, Hamada S. Inhibitory effect of oolong tea polyphenols on glycosyltransferases of mutans streptococci. Appl Environ Microbiol 1993; 59: 968-973.

51 Oliveira RAG, Lima EO, Vieira WL, Freire KRL, Trajano VN, Lima IO et al. Estudo da interferência de óleos essenciais sobre alguns antibióticos usados na clínica. Rev Bras Farmacogn. 2006; 16: 77-82.

52 Ooshima T, Minami T, Aono W, Izumitani A, Sobue S, Fujiwara T, Kawabata S, Hamada S. Oolong tea polyphenols inhibit experimental dental caries in SPF rats infected with mutans streptococci. Caries Res. 1993; 27: 124-129.

53 Ooshima T, Minami T, Matsumoto M, Fujiwara T, Sobue S, Hamada S: Comparison of the cariostatic effects between regimens to administer oolong tea polyphenols in SPF rats. Caries Res 1998; 32: 75-80.

54 Otake S, Makimura M, Kuroki T, Nishihara Y, Hirasawa M: Anticaries effects of polyphenolic compounds from Japanese green tea. Caries Res 1991; 25: 438-443.

55 Ozaki Y, Soedigdo S, Wattimena YR, Suganda AG. Anti-inflammatory effect of mace, aril of Myristica fragrans Houtt., and its active principles. Jpn. J. Pharmacol. 1989; 49: 155-163.

56 Paraskevas S: Randomized controlled clinical trials on agentes used for chemical plaque control. Int J Dent Hyg 2005; 3:162-178.

57 Park, S., Lee, D.K., Yang, C.H.,. Inhibition of fos-jun-DNA complex formation by dihydroguaiaretic acid and in vitro cytotoxic effects on cancer cells. Cancer Lett. 1998; 127: 23-28.

58 Quivey Jr. RG, Kuhnert WL, Hahn K. Adaptation of oral streptococci to low pH. Adv Microb Physiol. 2000; 42: 239-74.

59 Ribeiro JT, Costa MMNFG, Feitosa SVHS, Colares V. Avaliação da qualidade de vida de pré-escolares portadores de cárie severa. Arq Odontol, 2004; 40(2): 115-126.

61 Sawamura S, Tonosaki Y, Hamada S, Inhibitory effects of ellagic acid on glucosyltransferases from mutans streptococci. Biosci. Biotechnol. Biochem. 1992; 56: 766-768. 


\section{RECISATEC - REVISTA CIENTIFICA SAÚDE E TECNOLOGIA}

ALIMENTOS FUNCIONAIS NA CÁRIE DENTAL Julia das Neves Rodrigues Ferreira, Priscila Luiza Mello, Luiz Eduardo Nunes Ferreira

62 Scalbert A. Antimicrobial properties of tannins. Phytochemistry 1991; 30: 3875-3883.

63 Scalbert A, Manach C, Morand C, Rémésy C, Jiménez L. Dietary polyphenols and the prevention of diseases. Crit Rev Food Sci Nutr. 2005; 45(4):287.

64 Signoretto C, Canepari P, Stauder M, Vezzulli L, Pruzzo C. Functional foods and strategies contrasting bacterial adhesion. Curr Opin Biotechnol. 2012; 23(2): 160-167.

65 Simões M, Antimicrobial strategies effective against infectious bacteria biofilms. Current Medicinal Chemistry, vol. 18, no. 14, pp. 2129॰2145, 2011

66 Sköld-Larsson K, Fornell AC, Lussi A, Twetman S. Effect of topical applications of a chlorexidine/thymol-containing varnish on fissure caries assessed by laser fluorescence. Acta Odontol Scand 2004; 62:339-342.

67 Smullen J, Koutsou GA, Foster HA, Zumbé A, Storey DM. The antibacterial activity of plant extracts containing polyphenols against Streptococcus mutans. Caries Res. 2007; 41(5):342-9.

68 Socransky SS. Microbial agents and production of oral diseases.J Dent Res. 1968 NovDec;47(6):923-4.

69 Sonavane GS, Sarveiya VP, Kasture VS, Kasture SB,. Anxiogenic activity of Myristica fragrans seeds. Pharmacol. Biochem. Behav. 2002; 71: 239-244.

70 Souza AMPA. A influência das condições de trabalho na atividade de cárie dental de trabalhadores em padaria e confeitaria: estudo de caso, [dissertation], Florianópolis: UFSC, 2002, available in: http://www.nuppre.ufsc.br/wp-content/uploads/2/souzaana.pdf .

71 Tagashira M, Uchiyama K, Yoshimura T, Shirota M, Uemitsu N. Inhibition by hop bract polyphenols of cellular adherence and water-insoluble glucan synthesis of mutans streptococci. Biosci. Biotechnol. Biochem. 1997; 61: 332-335.

72 Thimothe J, Bonsi IA, Padilla-Zakour OI, Koo H. Chemical characterization of red wine grape (Vitis vinifera and Vitis interspecific hybrids) and pomace phenolic extracts and their biological activity against Streptococcus mutans. J Agric Food Chem. 2007; 55(25):10200-7.

73 Weyne SC. A construção do paradigma de promoção de saúde: um desafio para as novas gerações. In: Kriger L, coordenador. ABOPREV: promoção de saúde bucal. São Paulo: Artes Médicas; 1997. p.1-26.

74 Wu CD, Wei GX. Tea as a functional food for oral health. Nutrition. 2002; 18(5): 443-444.

75 Wu CD. Grape products and oral health. J Nutr. 2009; 139(9): 1818S-23S.

76 Yanagida A, Kanda T, Tanabe M, Matsudaira F, Oliveira CJG. Inhibitory effects of apple polyphenols and related compounds on cariogenic factors of mutans streptococci. J Agric Food Chem. 2000; 48(11):5666-71. 


\section{RECISATEC - REVISTA CIENTÍFICA SAÚDE E TECNOLOGIA}

ALIMENTOS FUNCIONAIS NA CÁRIE DENTAL Julia das Neves Rodrigues Ferreira, Priscila Luiza Mello, Luiz Eduardo Nunes Ferreira

77 Yam TS, Shah S, Hamilton-Miller JM: Microbiological activity of whole and fractionated crude extracts of tea (Camellia sinensis), and of tea components. FEMS Microbiol Lett 1997; 152: 169-174.

78 Yoshizumi AO, Liechocki DGL, Tanaka GY, Ferreira CFG, Benelli EM. Efeitos de diferentes nutrientes na estrutura do biofilme dentalformado in situ e in vitro por streptococcus mutans [Resumo]. Revista Dens. 2007; 15(2): 2. 